\title{
The effects of stimulus numerosity, retinal location, and rod contrast on perceived duration of brief visual stimuli
}

\author{
GERALD M. LONG and ROBERT J. BEATON \\ Villanova University, Villanova, Pennsylvania 19085
}

\begin{abstract}
The effects of manipulating three stimulus parameters were determined on a standard perceived duration task using 40- and 70-msec visual stimuli. In Experiment 1, it was found that perceived duration increased with increasing number of target dots but decreased as the area that contained the dots also increased. Experiment 2 examined the effect of varying the retinal location $(0,2$, or $4 \mathrm{deg}$ from fovea) of the target dot and found perceived target duration to increase with increasing eccentricity. In Experiment 3, the background luminance for a constant red target was varied so as to alter the scotopic contrast of the target. Perceived duration was minimal for the scotopically matched target and background conditions. The results of all three experiments were discussed in terms of important retinaland even rod-contributions to tasks of perceived duration.
\end{abstract}

A considerable number of recent studies have proposed that changes in the perceived duration of brief visual targets under specific stimulus manipulations may reveal the nature of information-processing mechanisms within the visual system (cf. Avant \& Lyman, 1975; Avant, Lyman, \& Antes, 1975; Thomas \& Cantor, 1975, 1976). However, criticism of this approach has recently arisen from two very different quarters. Gomez and Robertson (1979) and Robertson and Gomez (1980) have argued that variations in the nontemporal dimensions of a stimulus (e.g., size, complexity) may have an effect on duration estimates by altering the strategy or set established within the observer. They conclude that "non-temporal properties of each stimulus in isolation are insufficient to produce an illusion [of altering perceived duration]"' (Gomez \& Robertson, 1979, p. 437). As another alternative to the information-processing conceptualizations, Long and Beaton (1980a, 1980b) have proposed that several of the information manipulations in the perceived duration literature are interpretable in terms of the predictable effects upon retinal persistence. The purpose of this note is to present additional empirical support for this sensory persistence interpretation of perceived duration effects.

In previous research, Long and Beaton (1980a, $1980 \mathrm{~b}$ ) have demonstrated that changes in target size, target duration, target luminance, target contrast, and spatial frequency of the target all influence perceived duration in a manner predictable from

This research was supported by NIMHGrant No. 1 RO3MH3499801 to the first author. Reprint requests should be sent to Gerald M. Long, Department of Psychology, Villanova University, Villanova, Pennsylvania 19085. a consideration of the retinal effects of such manipulations. They argued that the most parsimonious explanation of these manipulations was then in terms of peripheral effects rather than the central processing stages favored by other investigators. In this article, the results of additional stimulus manipulations on tasks of perceived duration are presented. It is believed not only that the results are totally unexpected from an information-processing conceptualization of the task, but also that they explicitly reveal the role of retinal factors in task performance. Specifically, on a common perceived duration task, the effects of three manipulations were examined: (1) the number and density of multidot patterns comprising the brief target. (2) the retinal location of the target, and (3) the rod (scotopic) contrast of the target against its background.

\section{EXPERIMENT 1}

This experiment is concerned with the effects of stimulus numerosity and density on perceived duration. Previous investigators have reported that the perceived duration of a target increases as the number of elements comprising the target also increases (Buffardi, 1971; Mo, 1971, 1974, 1975). This effect was interpreted as support for the role of cognitive mechanisms in duration judgments. The stimuli containing the higher number of elements theoretically required a greater time to process. Alternatively, however, the effect of numerosity may be due to the increasing size and clarity of the fading iconic image produced by the compound stimulus. That is, increasing the number of stimulus elements within a constant size area would be expected to result in a more distinct sensory impression from that stimulus. 
As Long and Beaton (1980b) have previously shown, increasing the phenomenal "distinctness" (degree of filling) of a stimulus leads to subjectively longer iconic images. If this conceptualization of the numerosity effect is correct, then a critical related variable would be the size of the retinal area in which the target elements fall. This follows from a consideration of the relative density of the elements on the retina, which in turn would influence the phenomenal clarity of the target. Two brief target presentations of equal luminance and duration and containing an equal number of elements (dots) would not be expected to produce equal persistence if the areas in which the dots fall are very different. Experiment 1 tested this prediction.

\section{Method}

Subjects. Thirteen undergraduates (Seven males, six females) at Villanova University participated in this experiment for credit toward course requirements in general psychology. Subjects were run in individual 1 -h sessions.

Apparatus and Stimuli. A Scientific Prototype tachistoscope (Model 320-GB) was employed in all three experiments. The target stimuli in this experiment varied in the number of stimulus elements and the size of the area in which the elements were arranged. The stimulus elements were black squares, each subtending approximately $.3 \mathrm{deg}$. The target stimulus contained 1,4 , or 8 squares. For each level of numerosity, the squares were arranged in a

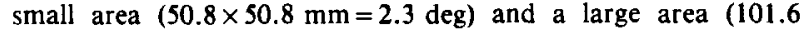
$\times 101.6 \mathrm{~mm}=4.7 \mathrm{deg})$. The stimulus configurations were determined by randomly choosing a position within each area in which to place a square. Thus, there were six ( 2 area by 3 numerosity) theoretically possible combinations. However, only five combinations of area and numerosity were actually used because the one-square condition was considered functionally equivalent for both the small and large areas. Three examples of each of the five numerosity-area conditions were constructed. The luminance of the white target background as well as the pre- and postexposure fields was $15 \mathrm{fL}\left(51.4 \mathrm{~cd} / \mathrm{m}^{2}\right)$. Target duration was either 40 or $70 \mathrm{msec}$.

Procedure. The perceived duration task devised by Thomas and Cantor $(1975,1976)$ was employed. Each experimental trial began with the observer's viewing the preexposure fixation field and receiving a verbal "ready" signal from the experimenter. After a 500-msec delay, the target field was presented for either 40 or $70 \mathrm{msec}$. The target field was immediately replaced by the blank postexposure fixation field. Following each stimulus presentation, the observers verbally reported whether perceived duration of the target was "short," "medium," or "long." The experimenter recorded the duration judgment and proceeded to the next trial condition.

The experimental session began with 10 practice trials in which the observer viewed all combinations of numerosity, area, and duration employed in this experiment. These trials were to familiarize the observers with the task and stimuli, but observers were not trained to call any particular stimulus condition "short," "medium," or "long." Following practice, the observers received 120 experimental trials consisting of 12 presentations of the 10 stimulus conditions. The order of the 10 stimulus conditions was uniquely randomized for each observer within the constraint that each stimulus condition occur every 10 trials and that each example of the numerosity-area manipulation occur every 30 trials. After 60 experimental trials, the observers were given a 2-3-min rest period. No feedback about the observers' responses was given at any time during the experiment.

\section{Results and Discussion}

The duration judgments of "short," "medium," and "long" were coded as 0,1 , and 2 , respectively, following the analysis employed by Gomez and Robertson (1979) and Thomas and Cantor (1975, 1976). The mean results for the 13 observers on the 10 stimulus conditions are shown in Figure 1. The mean estimates of perceived duration for the 40 and 70-msec targets as a function of number dotsquares in the target are presented for both the small (Figure 1a) and the large (Figure 1b) stimulus areas. Because the data points for the one-dot condition are based on the identical responses in the top and middle figures, two separate repeated-measures analyses of variance were computed so that the onedot conditions could be included in both. For the data in Figure 1a, the main effect of numerosity was highly significant $[\mathrm{F}(2,24=88.3, \mathrm{p}<.001]$, as was the main effect of the target duration $[F(1,12)$ $=13.0, \mathrm{p}<.005]$. The interaction was not significant $[F(2,24)=1.7, p>.20]$. The identical pattern of results was found for the data presented in Figure $1 \mathrm{~b}$. The main effect of numerosity was significant $[F(2,24)=18.2, p<.001]$, and the main effect of target duration was significant $[F(1,12)=35.2$, $p<$ $.001]$, but the interaction again failed to reach significance $[F(2,24)=2.5, p>.10]$. These results are

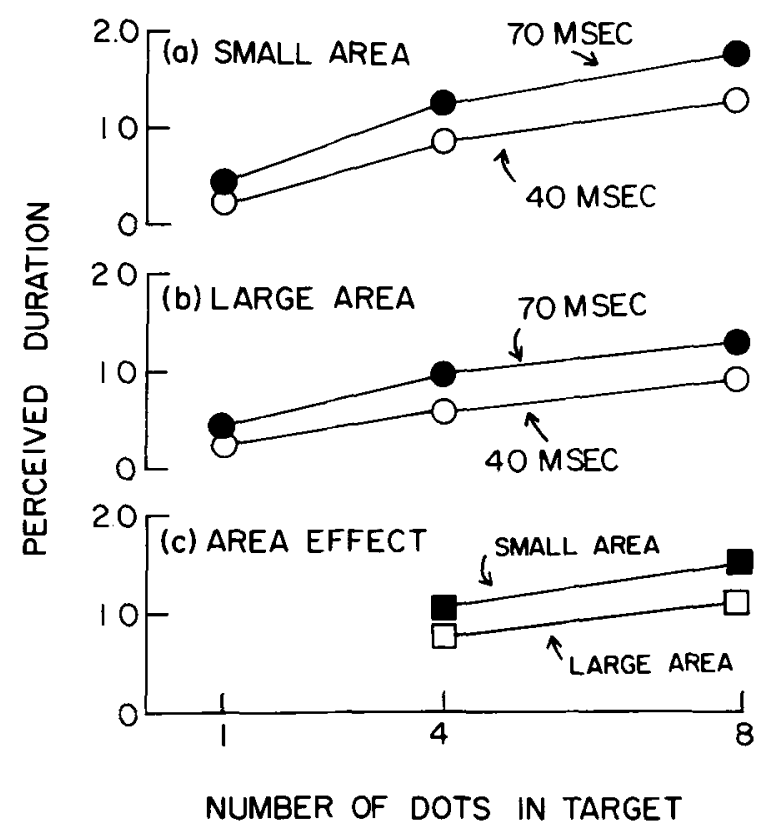

Figure 1. Mean estimates of perceived duration as a function of number of dots in target and size of area comprising 40and 70-msec targets in Experiment 1. In (a), the estimates are from the small area condition only; in (b), the estimates are from the large area condition. In (c), the two duration conditions for the 4- and 8-dot levels in (a) are combined and compared with the corresponding conditions in (b) to provide a comparison of area effects. 
essentially consistent with those reported by other investigators (e.g., Buffardi, 1971; Mo, 1971, 1974, 1975). However, the results presented in Figure 1c support the argument proposed here that retinal factors are extremely important in the effects obtained by manipulating stimulus parameters on perceived duration tasks. Figure 1c contains the combined results from Figures $1 \mathrm{a}$ and $1 \mathrm{~b}$ collapsed over the two durations so that the effect of retinal area can be assessed. (The data for the one-dot condition are deleted because, as described above, these conditions were identical in the two area manipulations.) The $2 \times 2$ repeated-measures analysis of variance for the data shown in Figure 1c revealed a significant main effect of numerosity $[F(1,12)=41.4$, $\mathrm{p}<.001]$ and, very importantly, target area $[\mathrm{F}(1,12)$ $=5.9, \mathrm{p}=.03]$. The interaction was not significant $[F(1,12)=3.9, p>.05]$. Hence, as predicted, the area in which the multiple elements comprising the target fall is important in duration estimates. It is believed that this results from the greater subjective retinal contrast produced by multiple stimuli in a small area than by the same number of stimuli in a large area.

\section{EXPERIMENT 2}

In the peripheral interpretation of visual persistence, it is proposed that the rod photoreceptors provide the bulk of the persistence (cf. Long, 1980; Sakitt, 1975, 1976; Sakitt \& Long, 1978, 1979a, 1979b). Given the well-known distributions of cones and reds on the retina (e.g., Brown, 1965), one would then predict some changes in the character of persistence as the location of the target on the retina is varied. And, indeed, Sakitt and Long (1978) have reported changes in the relative contribution to persistence of the cone and rod systems as retinal eccentricity of a target is varied from foveal to $10 \mathrm{deg}$ eccentric viewing. If, as the present argument suggests, perceived duration of brief targets reflects directly the degree of sensory persistence produced by a stimulus, then performance on a perceived duration task should also be sensitive to the retinal location of that target stimulus. Experiment 2 examines this proposal.

It should be clear from a consideration of the retinal variables involved that the effect of varying target eccentricity cannot be absolute. Two examples should demonstrate this point. First, as a target is moved from foveal to peripheral positions, visual acuity suffers; and it is known that clarity of the target can influence perceived duration (Avant, Lyman, \& Antes, 1975; Long \& Beaton, 1980a, $1980 \mathrm{~b})$. Second, in some common experimental conditions, the participation of the rod system is frequently minimized. For example, bright pre- and postexposure fields can significantly reduce rod contributions and may even totally saturate the rod system if the upper luminance limits of typical tachistoscopes are used (e.g., Sakitt, 1976; Sakitt \& Appelman, 1978). Hence, for the viewing manipulation of interest here, an effort was made to insure that the rod system was not excluded from participation on the task. Specifically, a relatively large luminous target was used, and the pre- and postexposure fields were kept dark. It should be noted, however, that such conditions are by no means unknown in time perception research (e.g., Allan, Kristofferson, \& Rice, 1974; Allan, Kristofferson, \& Wiens, 1971; Buffardi, 1971; Goldstone, Lhamon, \& Sechzer, 1978), and they were considered entirely appropriate for the purpose of demonstrating that retinal variables can influence perceived duration estimates.

\section{Method}

Subjects. Five undergraduates (two males, three females) at Villanova University participated in this experiment in return for credit toward fulfilling a general psychology requirement.

Stimuli. The target stimulus was a single 2-deg white dot positioned on a black background. The retinal eccentricity of the white dot was varied over three levels by positioning the dot at 0 , 2 , and $4 \mathrm{deg}$ to the right of the center of the background field. The luminance of the dot was constant at $5 \mathrm{fL}\left(17.1 \mathrm{~cd} / \mathrm{m}^{2}\right)$. The luminances of the dark background of the target and the dark pre- and postexposure fields were approximately $.83 \mathrm{fL}$ $\left(2.8 \mathrm{~cd} / \mathrm{m}^{2}\right)$. Target duration was either $\mathbf{4 0}$ or $70 \mathrm{msec}$.

Procedure. As in Experiment 1, the perceived duration task of Thomas and Cantor $(1975,1976)$ was employed. The observers were required to report "short," "medium," or "long." Following a practice session that familiarized the observer with the six stimulus conditions ( 2 durations by 3 eccentricities), the observers were presented 120 experimental trials. The order of the stimulus conditions was uniquely randomized for each observer within the single constraint that each stimulus condition occur every six trials. For each observer, 20 observations were obtained for each stimulus condition. After 60 trials, the observers were allowed a brief rest. Before the experiment, each observer darkadapted for approximately $15 \mathrm{~min}$.

\section{Results and Discussion}

The duration judgments of "short," "medium," and "long" were coded as 0,1 , and 2, respectively, as in Experiment 1. The mean responses for the five observers over the six stimulus conditions are shown in Figure 2. A repeated-measures analysis of variance indicated significant main effects of retinal eccentricity $[F(2,8)=18.6, p<.001]$ and target duration $[F(1,4)=24.8, p=.008]$; the interaction was not significant $[\mathrm{F}(2,8)=2.0, \mathrm{p}=.19]$.

The results shown in Figure 2 indicate an important effect of retinal location on the perceived duration of a brief target under the stimulus conditions employed here. These findings are entirely consistent with the proposal that retinal persistence effects may contribute to estimates of perceived duration in at least some situations. However, regardless of the basis of the effect, the findings presented here serve to caution in comparing or contrasting across studies that have not employed similar stimulus conditions. 


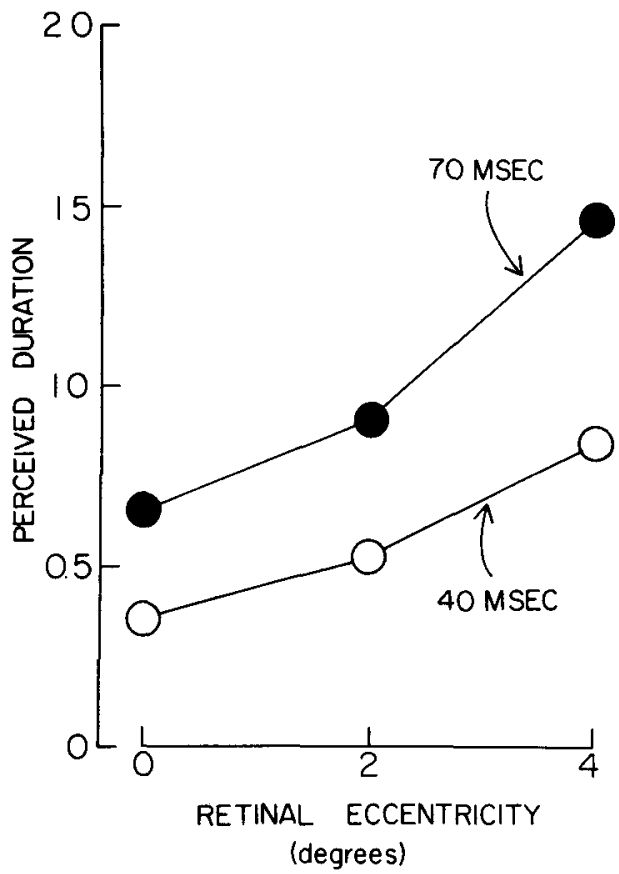

Figure 2. Mean estimates of perceived duration of 40- and 70-msec targets as a function of retinal location of the target. (Experiment 2)

\section{EXPERIMENT 3}

This final experiment attempted to demonstrate further the important role of retinal-and, specifically, rod-processes in judgments of brief visual durations. Although it was proposed that these processes underlay the findings of Experiment 2, it was noted that other variables, such as stimulus clarity, also necessarily change with increasing eccentricity, in addition to relative rod stimulation, which was of specific interest. In this experiment, a red target of constant size, luminance, and location is presented against three different background luminances. However, because the red target stimulus has the identical scotopic (rod) luminance as the middle-level background luminance used, minimal rod persistenceand, thereby, minimal perceived duration- is predicted for this condition.

This specific procedure of manipulating the scotopic contrast of a target is borrowed directly from the iconic memory literature (Adelson, 1978; Banks \& Barber, 1977; Sakitt \& Long, 1979b). With this procedure, it has been shown that the subjective persistence of a brief target is greatly reduced when rod contrast is minimized (Adelson, 1977; Sakitt \& Long, 1979b). If, as argued, this same persistence contributes to estimates of perceived duration, similar effects should result from this manipulation on a standard perceived duration task. Moreover, the effects should be much more pronounced under conditions in which rod participation is made more salient. Hence, the effect of varying the scotopic contrast of a target will be examined under both bright and dark pre- and postexposure conditions. A much more marked effect is predicted for the dark postexposure conditions, which permits greater contributions from the rod system.

\section{Method}

Subjects. Thirteen undergraduates (five males, eight females) at Villanova University participated in the experiment in return for partial fulfillment of a requirement in a general psychology course.

Stimuli. Three stimuli were constructed that varied in the degree of rod contrast between the target and the background. In order to establish a relative scotopic match (i.e., zero rod contrast), one of the authors (R.B.) first dark-adapted for $45 \mathrm{~min}$. Then, under scotopic lighting conditions in the tachistoscope, he chose a sheet of red colored paper (Color Aid, T-3) that appeared equal in achromatic brightness to a sheet of gray colored paper (Color Aid, Gray \#6). Two additional shades of gray paper (Color Aid, Gray \#4 and \#8), one much brighter and one much darker than the gray selected for the scotopic match, were chosen in order to construct negative and positive rod contrast stimuli. Circular stimuli $(2 \mathrm{deg})$ were constructed from the red paper and then centered on each of the three gray backgrounds. In the experimental sessions that followed, the photopic luminance of the red dot was constant at about $1.1 \mathrm{fL}\left(3.9 \mathrm{~cd} / \mathrm{m}^{2}\right)$, and the background luminances of the three gray cards varied over three levels: $1.4 \mathrm{fL}\left(4.8 \mathrm{~cd} / \mathrm{m}^{2}\right), .8 \mathrm{fL}\left(2.7 \mathrm{~cd} / \mathrm{m}^{2}\right)$, and $.2 \mathrm{fL}\left(.7 \mathrm{~cd} / \mathrm{m}^{2}\right)$. Target duration was either 40 or $70 \mathrm{msec}$. In addition to the three levels of rod contrast, there were two levels of luminance for the adapting field that preceded and followed the target presentation. These pre- and postexposure fields were either "dark," $.83 \mathrm{fL}$ $\left(2.8 \mathrm{~cd} / \mathrm{m}^{2}\right)$, or "light," $5.0 \mathrm{fL}\left(17.1 \mathrm{~cd} / \mathrm{m}^{2}\right)$. Six subjects were assigned randomly to the "dark" field conditions, and seven subjects were assigned randomly to the "light" field conditions.

Procedure. The basic procedure employed for this experiment was identical to that in Experiments 1 and 2. Observers were required to report verbally whether the duration for each stimulus presentation was "short," "medium," or "long." In a brief practice session, the observers were familiarized with all six possible stimulus conditions they would see in the experimental session, but they were not trained to designate the conditions with specific duration labels. In the experimental session that followed, all observers were presented 120 experimental trials. The six stimulus conditions (two durations and three backgrounds) were uniquely randomized for each subject within the constraint that each condition occur every six trials. Also, as in Experiment 2, observers were dark-adapted for 15 min prior to the experimental session.

\section{Results and Discussion}

The duration judgments were coded as in the two previous experiments. The mean data for the two adaptation fields are shown in Figure 3. Because observers' temporal judgments of each stimulus condition were relative judgments based upon the six particular conditions to which they were exposed, it is inappropriate to compare the absolute levels obtained in Figures 3a and 3b, which represent different observers. Hence, two repeated-measures analyses of variance were computed. For the "dark" field condition (Figure 3a), the main effect of background luminance was highly significant $[F(2,10)=23.6$, $p<.001]$, as was the main effect of target duration 

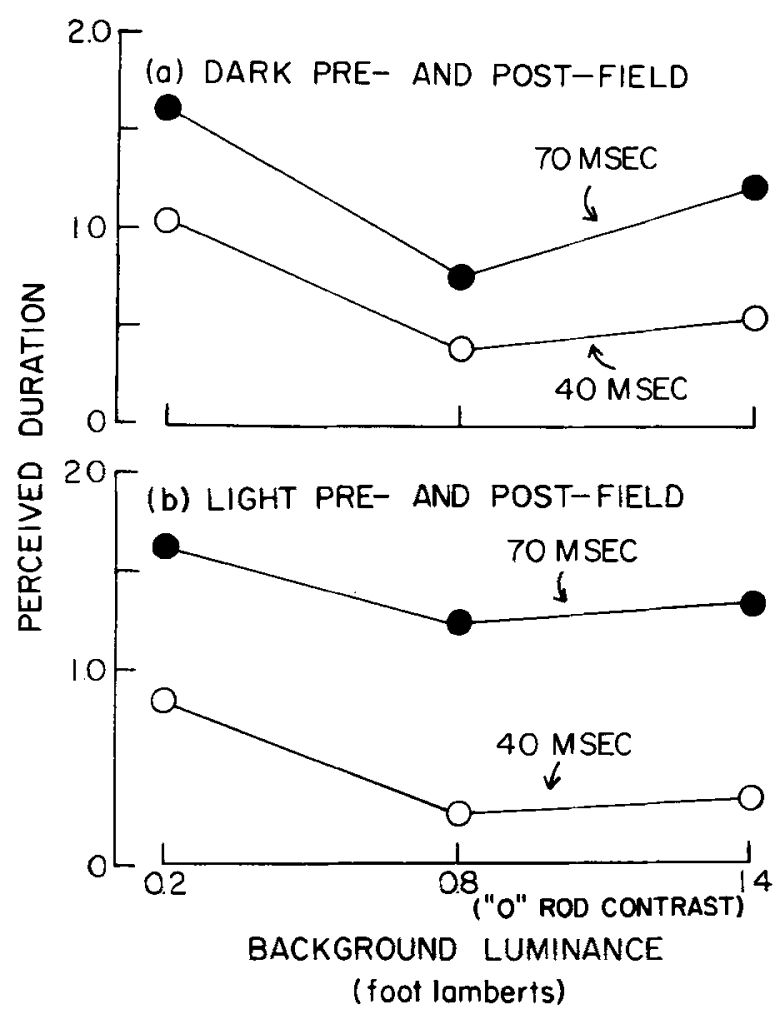

Figure 3. Mean estimates of perceived duration of 40- and 70-msec targets as a function of background level with dark (a) and light (b) adaptation fields. Note that, for the rod system, the .8-fL. background level is theoretically equivalent to the 1.1-fL red target. (Experiment 3)

$[F(1,5)=108.2, p<.001]$. The interaction was not significant $[F(2,10)=2.8, p>.10]$. It was predicted that the middle-level background luminance $(.08 \mathrm{fL})$ would produce minimal rod persistence and, consequently, minimal perceived duration. For the dark pre- and postfield conditions (Figure 3a), this was the case. A Newman-Keuls analysis indicated that the perceived durations for the .8 -fL background condition differed significantly from those obtained in both the .2 -fL condition ( $p<.01$ ) and the 1.4-fL condition $(p<.05)$. It is believed that this pattern of results reveals the importance of rod participation in visual persistence and, by extension, perceived duration. This interpretation is further supported by the results from the light pre- and postfield condition (Figure 3b). With light fields, participation of the rod system would be reduced as it rapidly approaches total saturation (Aguilar \& Stiles, 1954). Consequently, the persistence produced by a target under light field conditions would be primarily a cone phenomenon. Consequently, a manipulation of rod contrast of a target to its background would have much less of an effect. That is, photopic (cone) contrast would be the more critical variable. As shown in Figure 3b, the "V"'-shaped nature of the results in Figure 3a has largely disappeared when light pre- and postfields are used. An analysis of variance again indicated a significant main effect of background luminance $[F(2,12)=3.9, p<.001]$, as well as a significant main effect of target duration $[F(1,6)=212.0$, $\mathrm{p}<.001]$. The interaction was not significant $[\mathrm{F}(2,12)$ $=2.6, p>.10]$. Newman-Keuls analyses further revealed that the .2 -fL condition differs significantly from both the .8-fL and 1.4-fL conditions $(p<.01)$, but the latter two conditions do not differ from each other $(\mathrm{p}>.05)$.

\section{SUMMARY}

This article has attempted to provide additional support for the claim that judgments of the perceived duration of brief visual stimuli are significantly influenced by the relative amount of sensory persistence produced by the stimuli. This view stresses the importance of very early peripheral levels in the visual system that are sensitive to such basic parameters as luminance, duration, wavelength, adaptation level, contrast, size, etc. In this regard, the present study examined the effect on perceived duration of additional characteristics of the stimulus-specifically, the numerosity and density of multielement targets, the retinal location of a target, and the scotopic contrast of a target against its background. These particular conditions were chosen for two reasons. First, these variables would be predicted to affect the degree of retinal persistence produced by a stimulus. Second, the current information-processing models would seem to have special difficulty in accounting for changes in perceived duration produced by these specific variables. Alternately, a proposal based upon the role of cone and rod persistence effects (cf. Long, 1980; Sakitt, 1976) can quite readily incorporate these same results.

Experiment 1 demonstrated that perceived duration increased both with increasing numerosity of a multielement pattern and with decreasing area in which the elements were contained. It was argued that the critical manipulation in this experiment involved the phenomenal contrast of the target, which would increase as the number and density of target elements increased. Experiment 2 examined the effect of retinal location of a single target stimulus. It was found that, as retinal location varied from foveal to 2 to 4 deg eccentricity, the perceived duration of the brief luminous target increased. Increasing rod involvement was proposed as the basis for this effect. Finally, Experiment 3 manipulated the background luminance for a constant luminance red target. It was demonstrated that minimal perceived duration resulted from that background luminance that had the identical scotopic brightness as the red target. For this target and background condition, rod contrast was theoretically eliminated, which would serve to reduce the degree of sensory persistence for the 
target by removing any contribution from the rod system. The results of these three experiments provide substantial support for the claim that peripheral factors, as distiñct. from higher order informationprocessing stages, play an important role in estimates of perceived duration. Furthermore, it is proposed that rod and cone persistence effects are likely bases for these peripheral factors.

\section{REFERENCES}

Adelson, E. H. Iconic storage: The role of rods. Science, 1978, 201, 544-546.

Aguilar, M., \& Stiles, W. S. Saturation of the rod mechanism of the retina at high levels of stimulation. Optica Acta, 1954, 1, 59-65.

Allan, L. G., Kristofferson, A. B., \& Rice, M. E. Some aspects of perceptual coding of duration in visual duration discrimination. Perception \& Psychophysics, 1974, 15, 83-88.

Allan, L. G., Kristofferson, A. B., \& Wiens, E. W. Duration discrimination of brief light flashes. Perception \& Psychophysics, 1971, 9, 327-334.

Avant, L. L., \& Lyman, P. J. Stimulus familiarity modified perceived duration on pre-recognition visual processing. Journal of Experimental Psychology: Human Perception and Performance, 1975, 1, 205-213.

Avant, L. L., Lyman, P. J., \& Antes, J. R. Effects of stimulus familiarity upon judged visual duration. Perception \& Psychophysics, 1975, 17, 253-262.

Banks, W. P., \& Barber, G. Color information in iconic memory. Psychological Review, 1977, 84, 536-546.

Brown, J. L. The structure of the visual system. In C. H. Graham (Ed.), Vision and visual perception. New York: Wiley, 1965.

Buffardi, L. Factors affecting the filled-duration illusion in the auditory, tactual, and visual modalities. Perception \& Psychophysics, 1971, 10, 292-294.

Goldstone, S., Lhamon, W. T., \& Sechzer, J. Light intensity and judged duration. Bulletin of the Psychonomic Society, 1978, 12, 83-84.
Gomez, L. M., \& Robertson, L. C. The filled-duration illusion: The function of temporal and nontemporal set. Perception \& Psychophysics, 1979, 25, 432-438.

Long, G. M. Iconic memory: A review and critique of the study of short-term visual storage. Psychological Bulletin, 1980, 88, 785-820.

Long, G. M., \& Beaton, R. J. The contribution of visual persistence to the perceived duration of brief targets. Perception \& Psychophysics, 1980, 28, 422-430. (a)

Long, G. M., \& BeAton, R. J. The effects of spatial frequency and target type on perceived duration. Perception \& Psychophysics, 1980, 28, 413-421. (b)

Mo, S. S. Judgment of temporal duration as a function of numerosity. Psychonomic Science, 1971, 24, 71-72.

Mo, S. S. Comparative judgment of temporal duration as a function of numerosity. Bulletin of the Psychonomic Society, 1974, 3, 377-379.

Mo, S. S. Temporal reproduction of duration as a function of numerosity. Bulletin of the Psychonomic Society, 1975, 5, 165-167.

SAKITT, B. Locus of short-term visual storage. Science, 1975 , 190, 1318-1319.

SAK ITt, B. Iconic memory. Psychological Review,1976, 83, 257-276.

Sakitt, B., \& Appelman, J. B. The effects of memory load and the contrast of the rod signal on partial report superiority in a Sperling task. Memory \& Cognition, 1978, 6, 562-567.

SakitT, B., \& Long, G. M. Relative rod and cone contributions in iconic storage. Perception \& Psychophysics, 1978, 23, 527-536.

SakitT, B., \& Long, G. M. Cones determine subjective offset of a stimulus but rods determine total persistence. Vision Research, $1979,19,1439-1441$. (a)

Sakitr, B., \& Long, G. M. Spare the rod and spoil the icon. Journal of Experimental Psychology: Human Perception and Performance, 1979, 5, 19-30. (b)

Thomas, E. A. C., \& Cantor, N. E. On the duality of simultaneous time and size perception. Perception \& Psychophysics, $1975,18,44-49$.

Thomas, E. A. C., \& Cantor, N. E. Simultaneous time and size perception. Perception \& Psychophysics, 1976, 19, 353-360.

(Received for publication October 1980; revision accepted February 13,1981 .) 